\title{
AVALIAÇÃO DA CONCENTRAÇÃO TOTAL E DA FRAÇÃO BIOACESSÍVEL DE METAIS EM AMOSTRAS DE CASCA DE UVA DE CULTIVARES TANNAT E CABERNET SAUVIGNON POR MIP OES
}

\author{
Bruno Jacobs ${ }^{a}$, Daisa Hakbart Bonemanna, Camila Corrêa Pereira ${ }^{a}$, Alexander Ossanes de Souza ${ }^{a}$, Ana Claudia Beduhn \\ Luckow $^{\mathrm{a}}$, Meibel Teixeira Lisboa ${ }^{\mathrm{a}}$, Anderson Schwingel Ribeiro ${ }^{\mathrm{a}}$, Solange Cadore ${ }^{\mathrm{b}}$ e Adriane Medeiros Nunes ${ }^{\mathrm{a}, *, 10}$ \\ ${ }^{a}$ Centro de Ciências Químicas, Farmacêuticas e de Alimentos, Universidade Federal de Pelotas, 96050-500 Capão do Leão - RS, \\ Brasil \\ 'Instituto de Química, Universidade Estadual de Campinas, 13083-970 Campinas - SP, Brasil
}

Recebido em 08/09/2020; aceito em 18/12/2020; publicado na web em 03/02/2021

\begin{abstract}
EVALUATION OF THE TOTAL CONCENTRATION AND THE BIOACCESSIBLE FRACTION OF METALS IN SAMPLES OF GRAPE SKINS OF CULTIVARS TANNAT AND CABERNET SAUVIGNON BY MIP OES. This paper describes the evaluation of the total concentration of $\mathrm{Ba}, \mathrm{Bi}, \mathrm{Ca}, \mathrm{Cd}, \mathrm{Co}, \mathrm{Cr}, \mathrm{Cu}, \mathrm{Fe}, \mathrm{K}, \mathrm{Mn}, \mathrm{Pb}$ e $\mathrm{Zn}$ and the bioaccessible fraction of $\mathrm{Ba}, \mathrm{Cu}, \mathrm{Fe}, \mathrm{Mn}, \mathrm{Pb}$ and $\mathrm{Zn}$ in samples of grape skins from different cultivars (Vitis Viníferas Tannat and Cabernet Sauvignon) by MIP OES. For the determination of total concentration, acid decomposition with a reflux system adapted to the digestion tubes in the oxidizing medium of $\mathrm{HNO}_{3}$ and $\mathrm{H}_{2} \mathrm{O}_{2}$ were used. The accuracy of the method was verified by recovery tests, whose values ranged from 80 to $120 \%$, as well as through a comparative method in closed system. $\mathrm{Bi}, \mathrm{Cd}$ and $\mathrm{Cr}$ showed values for total content below the LOD or below the values acceptable for health while $\mathrm{Pb}$ is higher than $0.2 \mathrm{mg} \mathrm{kg}^{-1}$, for both cultivars. To evaluate the bioacessible fraction, the gastrointestinal system was simulated using an in vitro procedure, and its performance was assessed considering the bioaccessible fraction (supernatant) and the non-bioaccessible fraction, whose sum should be closed to the total concentration, and the results obtained were between 81 and $120 \%$. Both cultivars showed similar values for the bioaccessible fractions $(\sim 13.5-53 \%$ of the total content).
\end{abstract}

Keywords: grape skin; Tannat and Cabernet Sauvignon; metals; total concentration; bioaccessibility.

\section{INTRODUÇÃO}

Em todo mundo, milhões de toneladas de resíduos provenientes das atividades agroindustriais são gerados anualmente. Embora a maior parte seja biodegradável, esses resíduos necessitam de tempo para serem decompostos, podendo causar danos ao meio ambiente devido à inadequada forma de descarte. ${ }^{1,2}$

Dentre os inúmeros resíduos provenientes do agronegócio no Brasil destaca-se o setor vinícola, pelo alto volume gerado, de aproximadamente 20 a $30 \%$, comparado com o peso total. Esse resíduo é composto pelo engaço, bagaço (casca e sementes) e a borra resultante do processo fermentativo, o que torna este setor promissor para o reaproveitamento de substâncias naturais. ${ }^{1,3}$

A casca da uva representa, em média, $82 \%$ do peso do bagaço, ${ }^{4}$ sendo uma fonte de antocianidinas e antocianinas, que são corantes naturais e possuem propriedades antioxidantes, além de apresentar açúcares e macro e micronutrientes, ${ }^{5,6}$ sendo também uma excelente fonte de fibra alimentar, que corresponde a cerca de 50 a $60 \%$ da composição total das cascas. ${ }^{7}$

A produção de 100 litros de vinho tinto gera $25 \mathrm{~kg}$ de resíduos, dos quais $17 \mathrm{~kg}$ são de bagaço. Esses subprodutos, que são compostos majoritariamente por cascas de uva, podem ser utilizados na elaboração da farinha de bagaço de uva, a qual pode ser aplicada na elaboração de pães, biscoitos, barras de cereais e massas, o que a torna uma excelente alternativa para os diabéticos, pois o consumo de frutas in natura é restrito para essas pessoas, devido ao alto teor de açúcar presente. ${ }^{8-11}$

Com base nas propriedades funcionais das substâncias presentes nesses subprodutos, que se caracterizam por auxiliar no metabolismo humano, como fibras, vitamina $\mathrm{C}$ e a presença de compostos fenólicos, cresce o interesse das indústrias para a extração desses compostos com o objetivo de agregar valor aos produtos alimentícios, ajudando

*e-mail: adriane.mn@hotmail.com na melhoria do padrão alimentar da população, além de prevenir diversos tipos de doenças. ${ }^{7,11,12}$

De acordo com o Instituto Brasileiro do Vinho, ${ }^{13} \mathrm{em} 2019$, a safra de uva no estado do Rio Grande do Sul, Brasil, foi caracterizada por sua qualidade, tendo sido colhidos 614,3 milhões de quilos de uvas destinados ao processamento de produtos vinícolas. Assim, devido ao grande volume de resíduos gerados e a importância relacionada às questões de sustentabilidade e problemas de caráter ambiental, cresce ainda mais a demanda por novas perspectivas que possibilitem o reuso desses subprodutos oriundos das indústrias vinícolas..$^{11,14,15}$

Tendo como base o contexto da reutilização destes resíduos pelos sistemas agrícolas familiares, bem como aplicados às mais diversas áreas industriais, o conhecimento da composição e a concentração dos constituintes inorgânicos presentes em amostras de casca de uva se faz necessário. Alguns desses constituintes possuem características fundamentais para o funcionamento do organismo, porém, quando em altas concentrações, podem vir a ser prejudiciais à saúde. Dessa forma, podem ser classificados como elementos essenciais, em concentração de gramas (Ca, K, Mg, N, Na, P e S); elementos traço, necessários na ordem de miligrama $(\mathrm{Cu}, \mathrm{Fe}, \mathrm{Mn}$ e $\mathrm{Zn})$; elementos ultra traço, necessários na ordem de micro a nanograma $(\mathrm{Se}, \mathrm{B}, \mathrm{Li}$, entre outros). ${ }^{16-18}$

No entanto, sabe-se que a determinação dos teores totais dos nutrientes presentes em uma determinada matriz alimentícia, nem sempre é capaz de estabelecer a real contribuição que eles podem nos proporcionar. Dessa forma, estudos de bioacessibilidade vêm contribuindo de forma significativa em análises químicas, os quais permitem conhecer a quantidade do composto ou elemento que é liberado da matriz da amostra, sendo solúvel no trato gastrointestinal, tornando-se disponível para absorção pelo epitélio intestinal. ${ }^{16,19,20}$ Para o estudo de bioacessibilidade é necessário conhecer o sistema gastrointestinal humano, além de entender que a amostra, quando ingerida, passa por um processo complexo que a transforma através de alterações mecânicas e enzimáticas, possibilitando que seus analitos 
possam ser absorvidos para entrarem na corrente sanguínea. Sendo assim, a primeira transformação da amostra ocorre na boca, seguida do estômago. Já a digestão enzimática, a absorção dos nutrientes e da água ocorrem predominantemente no intestino delgado e grosso. Existem diferentes trabalhos na literatura que relatam estudos de bioacessibilidade, porém, ainda não se tem um protocolo padronizado para a quantificação dos analitos bioacessíveis. Dessa forma, Minekus et al. ${ }^{21}$ no intuito de obter um consenso e com uma rede internacional formada por mais de 200 cientistas de 32 países, propôs um método de digestão estática padronizada e prática, com base em condições fisiologicamente relevantes e que podem ser aplicadas para vários parâmetros, os quais podem ser alterados para adaptarem-se às necessidades mais específicas, dependendo do alimento estudado. Por esse motivo, este artigo se baseou no trabalho desses pesquisadores para os procedimentos de bioacessibilidade. ${ }^{22-25}$

Dentre as diversas técnicas reportadas na literatura para determinação da concentração de elementos em amostras de alimentos, pode ser citada a técnica de espectrometria de emissão óptica com plasma induzido por micro-ondas (MIP OES). Uma técnica multielementar que apresenta baixo custo operacional, uma vez que a manutenção do plasma se dá por meio do gás nitrogênio, o qual é convertido por meio de um gerador de nitrogênio que capta o ar atmosférico. Diversos trabalhos são relatados na literatura utilizando essa técnica na determinação elementar de diferentes matrizes alimentares. ${ }^{17-18,20,26-28}$

Sendo assim, o presente trabalho tem como objetivo a avaliação da concentração total e da fração bioacessível de metais em amostras de casca de uva das cultivares Tannat e Cabernet Sauvignon, utilizando a técnica multielementar de MIP OES, a qual irá contribuir para uma melhor avaliação dessa matriz, obtendo informações para que auxilie em sua reutilização.

\section{PARTE EXPERIMENTAL}

\section{Equipamentos e parâmetros instrumentais}

Para a determinação multielementar das concentrações de $\mathrm{Ba}, \mathrm{Bi}$, $\mathrm{Ca}, \mathrm{Cd}, \mathrm{Co}, \mathrm{Cr}, \mathrm{Cu}, \mathrm{Fe}, \mathrm{K}, \mathrm{Mn}, \mathrm{Pb}$ e $\mathrm{Zn}$ nas amostras de casca de uva foi utilizado o espectrômetro de emissão óptica com plasma induzido por micro-ondas (MIP OES) modelo 4200 (Agilent Technologies, Austrália), equipado com um detector CCD (do inglês, charge-coupled device), monocromador Czerny-Turner com distância focal de $600 \mathrm{~mm}$, com uma faixa de comprimento de onda de $178-780 \mathrm{~nm}$ e sistema de introdução de amostras multimodal. O nitrogênio gasoso $\left(\mathrm{N}_{2}\right)$ utilizado para operação do plasma foi obtido através de um gerador de nitrogênio, modelo 4107 (Agilent Technologies), que produz $\mathrm{N}_{2}$ com pureza superior a 99,5\%. As determinações foram feitas na posição axial.

Todas as medidas foram feitas em triplicata com um tempo de integração de três segundos e velocidade de rotação da bomba peristáltica de $15 \mathrm{rpm}$. Os sinais de fundo foram corrigidos de forma automática pela subtração entre os espectros do branco e das amostras. As condições operacionais do MIP OES estão apresentadas na Tabela $1 \mathrm{~S}$.

As amostras de casca de uva passaram por um processo de homogeneização em um liquidificador ( $400 \mathrm{~W}$ de potência, Arno, Brasil). Em seguida, foram pesadas utilizando uma balança analítica com resolução de 0,1 mg e tara máxima de $210 \mathrm{~g}$ (Modelo AR 2140, Ohaus Adventurer, EUA). Para a etapa de decomposição ácida das amostras foi utilizado um bloco digestor convencional (Modelo MA-4025, Marconi, Brasil). Em cada tubo digestor de vidro foi acoplado um sistema de refluxo para evitar perdas por volatilização de analitos e reagentes. O sistema consiste em um tubo de vidro fechado preenchido com água, que é recirculada a uma temperatura de $15{ }^{\circ} \mathrm{C}$, controlada através de um banho termostatizado (Modelo Q214S2, Quimis, Brasil). ${ }^{29}$

Para o estudo da fração bioacessível foi utilizado um pHmetro (Modelo PHS-3B, PHTek, Brasil), um banho Dubnoff com aquecimento e agitação (Modelo Q226M2, Quimis, Brasil) e uma centrífuga Eppendorf (Modelo 5804 R, Alemanha).

\section{Materiais e reagentes}

Os reagentes utilizados foram de grau analítico e as soluções empregadas foram preparadas com água desionizada, obtida por um sistema de purificação de água Direct-Q 3 (Millipore Corporation, EUA), com resistividade de $18,3 \mathrm{M} \Omega \mathrm{cm}$. Ácido nítrico $65 \%(\mathrm{v} / \mathrm{v})$ (Synth, Brasil) foi purificado através de um sistema de sub-ebulição de quartzo (MA-075, Marconi, Brasil). Antes do uso, os materiais de vidro foram lavados e imersos em um banho de $\mathrm{HNO}_{3} 10 \%(\mathrm{v} / \mathrm{v})$ por um período de 48 horas, enxaguados com água desionizada e secos a temperatura ambiente.

As soluções padrão dos analitos foram preparadas diariamente em meio ácido a partir de uma solução estoque contendo $100 \mathrm{mg} \mathrm{L}^{-1}$ para $\mathrm{Ba}, \mathrm{Cd}, \mathrm{Co}, \mathrm{Cr}, \mathrm{Cu}, \mathrm{Mn}, \mathrm{Mo}, \mathrm{Pb}$ e $\mathrm{Zn}$ (Sigma-Aldrich, EUA), $10.000 \mathrm{mg} \mathrm{L}^{-1}$ para K e Fe (Merck, Alemanha) e $1000 \mathrm{mg} \mathrm{L}^{-1}$ para $\mathrm{Ca}$ (Merck, Alemanha). Para o método de decomposição ácida, foram utilizados $\mathrm{H}_{2} \mathrm{O}_{2} 30 \%$ (v/v) (Dinâmica, Brasil), $\mathrm{HNO}_{3} 65 \%$ (v/v) bidestilado (Neon, Brasil) e pérolas de vidro. Para a geração química de vapor, foram utilizadas soluções de $\mathrm{NaBH}_{4} 0,5 \%(\mathrm{~m} / \mathrm{v})$ estabilizada em meio alcalino com $\mathrm{NaOH} 0,5 \%(\mathrm{~m} / \mathrm{v})$.

Os reagentes utilizados para os estudos de bioacessibilidade foram: $\mathrm{KCl}, \mathrm{NaCl}, \mathrm{KH}_{2} \mathrm{PO}_{4}, \mathrm{MgCl}_{2}\left(\mathrm{H}_{2} \mathrm{O}\right)_{6}$ (Synth, Brasil), $\mathrm{NaHCO}_{3}$ e $\mathrm{HCl}$ (Merck, Alemanha), $\left(\mathrm{NH}_{4}\right)_{2} \mathrm{CO}_{3}$ (J.T. Baker, EUA), $\alpha$-Amilase da Aspergillus oryzae (Sigma 86250, China), Pepsina (Sigma P7000, Suíça), Bile (Sigma B8631, Nova Zelândia) e Pancreatina 3x (Sigma P1625, EUA).

Para a pesagem das amostras o meio de suporte utilizado foi papel de cigarro transparente, cem por cento $(100 \%)$ celulose (aLeda, Brasil).

\section{Preparo das amostras}

As uvas das cultivares Tannat e Cabernet Sauvignon foram doadas pela vinícola Guatambu, localizada no município de Dom Pedrito, Rio Grande do Sul, Brasil. Foram coletados aproximadamente $1000 \mathrm{~g}$ de locais diferentes do vinhedo, de forma a ter uma maior representatividade. Ambas as cultivares encontram-se na mesma propriedade localizada no interior do município, as quais possuem a seguinte localização geográfica: Tannat (Latitude: -30.98811597843 Sul, -54.492673088443 Oeste) e Cabernet Sauvignon (Latitude: -30.9855753741855 Sul, -54. 4943688125776 Oeste).

Após a etapa de coleta, as uvas foram lavadas com água ultrapura, Milli-Q, e posteriormente foi feito o desengace manual, separando a casca da polpa e da semente. Em seguida, a polpa foi prensada manualmente a fim de se obter o mosto. As cascas, juntamente com o mosto, foram adicionadas em frascos de vidro para simular o processo de fermentação e, após quatro dias, foram separadas, trituradas e armazenadas em freezer doméstico, em frascos de vidro com tampa rosca.

Para o estudo o teor de água (umidade) foram pesados aproximadamente $5 \mathrm{~g}$ das diferentes amostras diretamente em béqueres, os quais foram posteriormente levados ao aquecimento em estufa a uma temperatura de aproximadamente $105{ }^{\circ} \mathrm{C}$. Esse processo foi realizado até as amostras adquirirem peso constante. A porcentagem de umidade encontrada nas amostras foi de aproximadamente $40 \%$." 


\section{Decomposição ácida em bloco digestor com sistema de refluxo - método proposto}

Cerca de 2,5 g de amostra foram pesados em triplicata, o que corresponde a $1 \mathrm{~g}$ de massa seca. A amostras foram pesadas diretamente em papel de cigarro $100 \%$ celulose, sendo este devidamente fechado e inserido no tubo de digestão. O procedimento de decomposição seguiu o descrito no trabalho de Pereira et al.,${ }^{16}$ baseado no trabalho de Minekus et al. ${ }^{21}$ Sendo assim, foram adicionados $5 \mathrm{~mL}$ de $\mathrm{HNO}_{3}$ $65 \%$ (v/v) em cada tubo de digestão, na presença de pérolas de vidro, e aos tubos foi adaptado o sistema de refluxo utilizando uma recirculação de água a $15{ }^{\circ} \mathrm{C}$. O bloco de digestão foi submetido ao programa de aquecimento descrito na Tabela 1.

Tabela 1. Programa de aquecimento do bloco de digestão

\begin{tabular}{ccc}
\hline Etapa & Tempo $(\mathrm{min})$ & Temperatura $\left({ }^{\circ} \mathrm{C}\right)$ \\
\hline 1 & 10 & 80 \\
2 & 10 & 150 \\
3 & 120 & 200 \\
4 & 60 & 150 \\
\hline
\end{tabular}

Após a temperatura do bloco baixar para $150{ }^{\circ} \mathrm{C}$ e as amostras esfriarem a temperatura ambiente, na etapa 4, foi adicionado $1 \mathrm{~mL}$ de $\mathrm{H}_{2} \mathrm{O}_{2} 30 \%$ (v/v), e os tubos retornaram ao bloco digestor por mais 60 min. Após o resfriamento da solução, o volume foi transferido para frascos de polipropileno (PP) e avolumado a $20 \mathrm{~mL}$ com água desionizada.

\section{Decomposição ácida em sistema fechado de alta pressão - método comparativo}

Para validar o método proposto neste estudo, foi utilizado um sistema fechado com frascos de politetrafluoretileno (PTFE) em bloco digestor. A análise foi feita em triplicata, transferindo para os frascos aproximadamente $250 \mathrm{mg}$ de amostra, que corresponde a $100 \mathrm{mg}$ de massa seca da amostra, na presença de $2,5 \mathrm{~mL}$ de $\mathrm{HNO}_{3}$ $65 \%$ (v/v). Em seguida, os frascos foram fechados e colocados no bloco de digestão por 3 horas, a $140{ }^{\circ} \mathrm{C}$. Depois de esfriar, a solução resultante foi transferida para frascos de PP e diluída a $20 \mathrm{~mL}$ com água desionizada, para posterior leitura no MIP OES.

\section{Fração bioacessível}

$\mathrm{O}$ procedimento para a avaliação da bioacessibilidade in vitro foi adaptado de Minekus et al.,${ }^{21}$ com três etapas consecutivas, que representam o sistema digestivo humano: boca, estômago e intestino. Para o desenvolvimento das respectivas etapas foram preparadas as soluções dos sais que simulam as substâncias ativas do sistema digestivo (saliva, suco gástrico e suco intestinal) (Tabela 2S). Para simular o sistema digestivo, foram adicionados as enzimas e os sais, conforme apresentado na Tabela 2.

Para a avaliação da fração bioacessível, foram pesados, separadamente, $5 \mathrm{~g}$ de cada amostra, em triplicata, diretamente em frascos de PP de $50 \mathrm{~mL}$. Posteriormente, foi realizada a simulação da etapa da boca, adicionando $4 \mathrm{~mL}$ da solução de saliva sintética e $1 \mathrm{~mL}$ de $\mathrm{CaCl} 7,5 \mathrm{mmol} \mathrm{L}{ }^{-1}$. O pH foi ajustado para $7 \mathrm{com}$ $\mathrm{NaOH} 1 \mathrm{~mol} \mathrm{~L}^{-1}$ e colocado em banho Dubnoff com agitação e aquecimento, a $37^{\circ} \mathrm{C}$, por 10 minutos. Na segunda etapa, foram adicionados $9,1 \mathrm{~mL}$ da solução de suco gástrico, $700 \mu \mathrm{L}$ de $\mathrm{CaCl}$ $2 \mathrm{mmol} \mathrm{L}^{-1}$ e ajustou-se o $\mathrm{pH}$ para $3 \mathrm{com} \mathrm{HCl} 1 \mathrm{~mol} \mathrm{~L}^{-1}$. Os frascos com a solução foram colocados no banho Dubnoff, a $37^{\circ} \mathrm{C}$, por
2 horas. Na terceira etapa, foram adicionados aos frascos $18,5 \mathrm{~mL}$ da solução do suco intestinal, $1,35 \mathrm{~mL}$ de $\mathrm{CaCl} 9 \mathrm{mmol} \mathrm{L}^{-1}$ e o pH foi ajustado para 7. A solução foi novamente encaminhada para o banho Dubnoff, por 2 horas, a $37^{\circ} \mathrm{C}$.

Logo após, as amostras foram submetidas a um banho de gelo por 20 minutos para inativação enzimática. Posteriormente, foram centrifugadas por 20 minutos a $10.000 \mathrm{rpm}$ para separar a fase sólida (fração não bioacessível) do sobrenadante. Para determinação da fração bioacessível, o sobrenadante foi diluído para $15 \mathrm{~mL}$.

Tabela 2. Reagentes utilizados no preparo das soluções para simular o sistema digestivo

\begin{tabular}{lccc}
\hline Reagentes & $\begin{array}{c}\text { Amylase } \\
\text { Volume final: } \\
50 \mathrm{~mL}\end{array}$ & $\begin{array}{c}\text { Pepsina } \\
\text { Volume final: } \\
100 \mathrm{~mL}\end{array}$ & $\begin{array}{c}\text { Pancreatina + Bile } \\
\text { Volume final: } \\
200 \mathrm{~mL}\end{array}$ \\
\hline Enzima & $1,24 \mathrm{~g}$ & $2,2 \mathrm{~g}$ & $0,536 \mathrm{~g}+5,4 \mathrm{~g}$ \\
$\mathrm{KCl}$ & $1,5 \mathrm{~mL}$ & $1,4 \mathrm{~mL}$ & $2,72 \mathrm{~mL}$ \\
$\mathrm{KH}_{2} \mathrm{PO}_{4}$ & $750 \mu \mathrm{L}$ & $36 \mu \mathrm{L}$ & $640 \mu \mathrm{L}$ \\
$\mathrm{NaHCO}_{3}$ & $675 \mu \mathrm{L}$ & $2,5 \mathrm{~mL}$ & $17 \mathrm{~mL}$ \\
$\mathrm{NaCl}$ & -------- & $2,35 \mathrm{~mL}$ & $3,85 \mathrm{~mL}$ \\
$\mathrm{MgCl}_{2}\left(\mathrm{H}_{2} \mathrm{O}\right)_{6}$ & $100 \mu \mathrm{L}$ & $160 \mu \mathrm{L}$ & $880 \mu \mathrm{L}$ \\
$\left(\mathrm{NH}_{4}\right)_{2} \mathrm{CO}_{3}$ & $25 \mu \mathrm{L}$ & $400 \mu \mathrm{L}$ & ---------- \\
$\mathrm{HCl} \mathrm{1M}$ & $60 \mu \mathrm{L}$ & --------- & --------- \\
$\mathrm{HCl} 6 \mathrm{M}$ & --------- & $260 \mu \mathrm{L}$ & $280 \mu \mathrm{L}$ \\
\hline
\end{tabular}

\section{Fração não bioacessível}

Para avaliar a eficiência e a exatidão do estudo de bioacessibilidade, a fração não bioacessível foi analisada, sendo que a soma das duas frações deve corresponder à concentração total dos elementos, conforme descrito por Souza et al. ${ }^{20}$

Para esse procedimento, aproximadamente 2,5 $\mathrm{g}$ das frações sedimentadas após a centrifugação foram pesadas em papel para cigarro, em triplicata, e submetidas ao processo de decomposição ácida para posterior determinação dos elementos por MIP OES.

\section{RESULTADOS E DISCUSSÃO}

\section{Parâmetros de mérito}

As Tabelas 3S e 4S apresentam os parâmetros de mérito para a quantificação total dos analitos presentes nas amostras de casca de uva e da fração bioacessível, respectivamente, determinados por MIP OES. Foram obtidos bons coeficientes de correlação linear ao quadrado para todos os elementos $\left(\mathrm{R}^{2}>0,99\right)$ em uma faixa linear para o $\mathrm{K}$ variando de 0,525 a $5,2 \mathrm{mg} \mathrm{L}^{-1}$ e 0,025 a $0,2 \mathrm{mg} \mathrm{L}^{-1}$ para os demais elementos. Os limites de quantificação do método $\left(\mathrm{LQ}_{(\mathrm{m})}\right)$ e de detecção do método $\left(\mathrm{LD}_{(\mathrm{m})}\right)$ obtidos para o método proposto foram menores na determinação da concentração total, exceto para o Zn, o que era esperado, considerando-se a complexidade da matriz utilizada para simular o sistema gastrointestinal humano, que afeta a sensibilidade das medidas analíticas.

\section{Avaliação da exatidão}

Para avaliar a exatidão do método proposto foram feitos testes de recuperação dos analitos em três diferentes níveis de concentrações, obtendo-se recuperações que variaram de 80 a $120 \%$. Os valores de desvio padrão relativo (RSDs) foram inferiores a $10 \%$ para todos os 
elementos, comprovando a exatidão do método proposto, bem como uma precisão adequada, conforme apresentado na Tabela 5S.

A Tabela 6S mostra os resultados obtidos no experimento para verificar a exatidão do método de preparo das amostras proposto, utilizando o método de decomposição da amostra de casca de uva da cultivar Tannat em um sistema fechado. Para os resultados obtidos foi aplicado o teste estatístico $t$ Student pareado para um limite de confiança de $95 \%$, observando-se que não há diferenças significativas entre os resultados encontrados na decomposição das amostras com sistema de refluxo em relação à decomposição em sistema fechado em bombas de PTFE.

Dessa forma, é possível verificar que o sistema de decomposição ácida com refluxo foi eficiente na decomposição das amostras de casca de uva, possibilitando a subsequente determinação dos analitos.

\section{Determinação da concentração total}

Os resultados da determinação da concentração total dos elementos nas duas cultivares de casca de uva estão apresentados na Tabela 3. É possível observar que para Bi e Cd as concentrações foram inferiores ao $\mathrm{LD}_{(\mathrm{m})}$ para ambas as amostras. Por outro lado, a amostra Tannat apresentou concentração inferior ao $\mathrm{LD}_{(\mathrm{m})}$ para o $\mathrm{Cr}$, enquanto a concentração desse elemento na amostra Cabernet Sauvignon é inferior ao consumo diário recomendado. ${ }^{19,30}$

A cultivar Tannat apresentou as maiores concentrações de Ba, $\mathrm{Cu}, \mathrm{Fe}, \mathrm{K}, \mathrm{Mn}$ e $\mathrm{Pb}$ enquanto a cultivar Cabernet Sauvignon tem as maiores concentrações de $\mathrm{Ca}$, Co e $\mathrm{Zn}$. Embora as duas cultivares tenham sido produzidas na mesma localidade e no mesmo solo, recebendo o mesmo tipo de tratamento, foi possível observar que há uma diferença na absorção dos nutrientes por parte das videiras. De acordo com a literatura, o mesmo comportamento de absorção dos minerais por diferentes cultivares, plantadas no mesmo solo e nas mesmas condições, foi relatado por Ko et al. ${ }^{31}$

As altas concentrações de $\mathrm{K}$ encontradas nas amostras de casca de uva também foram relatadas no trabalho publicado por Mpelasoka et al. ${ }^{32}$ onde os autores mencionaram que as maiores quantidades deste elemento estão concentradas na casca, seguido das sementes e da polpa, sendo este elemento transportado via xilema e floema, sendo esta última via a principal entrada de $\mathrm{K}$ para a baga.

No trabalho desenvolvido por Yang et al. ${ }^{33}$ os autores avaliaram a concentração de diversos elementos na casca, polpa e sementes em três diferentes cultivares, entre elas a Cabernet Sauvignon, que apresentou concentrações de 3,0; 2,5; 8,0 e 1,5 mg kg-1 para os analitos $\mathrm{Zn}, \mathrm{Cu}, \mathrm{Fe}$ e $\mathrm{Mn}$ presentes na casca, respectivamente, valores muito próximos aos encontrados no presente trabalho. Assim, a composição mineral da casca para esses elementos mostra a existência de um padrão, já que são micronutrientes que auxiliam no desenvolvimento da planta. ${ }^{34}$

De acordo com a RDC No 42 - ANVISA, que dispõe sobre os limites máximos de contaminantes inorgânicos em alimentos, o limite para $\mathrm{Pb}$ é de $0,2 \mathrm{mg} \mathrm{kg}^{-1}$ e para o Cd é de $0,05 \mathrm{mg} \mathrm{kg}^{-1}$ para frutas frescas de bagos. ${ }^{35}$ Desta forma, a concentração total de $\mathrm{Pb}$ encontrada para as cultivares analisadas ultrapassa o limite permitido, o que demonstra a necessidade de se ter maior controle em relação aos níveis de contaminantes inorgânicos.

Para a Organização Mundial de Saúde, bem como para a Organização das Nações Unidas para a Alimentação e a Agricultura (OMS/FAO), através da Comissão Codex Alimentar, ${ }^{36}$ o limite máximo de $\mathrm{Pb}$ é de $0,1 \mathrm{mg} \mathrm{kg}^{-1}$, o que tornaria ainda mais preocupante a concentração encontrada. Já o Cd apresentou concentrações inferiores ao $\mathrm{LQ}_{(\mathrm{m})}$ que é $0,045 \mathrm{mg} \mathrm{kg}^{-1}$, não apresentando riscos de toxicidade ao consumidor para este elemento.
Tabela 3. Concentração total dos analitos nas amostras de casca de uva das cultivares Tannat e Cabernet Sauvignon obtidas por MIP OES

\begin{tabular}{ccc}
\hline \multirow{2}{*}{ Analito } & \multicolumn{2}{c}{ Concentração $\left(\mathrm{mg} \mathrm{kg}^{-1}\right)$} \\
\cline { 2 - 3 } & Tannat & Cabernet Sauvignon \\
\hline $\mathrm{Ba}$ & $5,72 \pm 0,22(3,8)$ & $2,36 \pm 0,07(3,0)$ \\
$\mathrm{Bi}$ & $<0,0086$ & $<0,0086$ \\
$\mathrm{Ca}$ & $105 \pm 3(2,8)$ & $132 \pm 5(3,8)$ \\
$\mathrm{Cd}$ & $<0,045$ & $<0,045$ \\
$\mathrm{Co}$ & $0,79 \pm 0,01(1,3)$ & $1,13 \pm 0,05(4,4)$ \\
$\mathrm{Cr}$ & $<0,0043$ & $0,0129 \pm 0,0008(6,2)$ \\
$\mathrm{Cu}$ & $3,59 \pm 0,15(4,2)$ & $2,39 \pm 0,20(8,4)$ \\
$\mathrm{Fe}$ & $5,9 \pm 0,3(5,1)$ & $5,5 \pm 0,5(9,1)$ \\
$\mathrm{K}$ & $3991 \pm 105(2,6)$ & $3495 \pm 130(3,7)$ \\
$\mathrm{Mn}$ & $2,21 \pm 0,20(9,0)$ & $1,92 \pm 0,07(3,6)$ \\
$\mathrm{Pb}$ & $0,51 \pm 0,3(5,9)$ & $0,25 \pm 0,01(4,0)$ \\
$\mathrm{Zn}$ & $1,72 \pm 0,03(1,7)$ & $3,35 \pm 0,19(5,7)$ \\
\hline
\end{tabular}

Média \pm desvio padrão (RSD).

\section{Resultados da determinação da fração bioacessível nas amostras de casca de uva}

Para verificar o que de fato pode ser aproveitado por nosso organismo em termos de nutrientes e elementos potencialmente tóxicos nas amostras de casca de uva analisadas, foi avaliada a bioacessibilidade de $\mathrm{Ba}, \mathrm{Cu}, \mathrm{Fe}, \mathrm{Mn}, \mathrm{Pb}$ e $\mathrm{Zn}$ em ambas as cultivares. É importante salientar que os elementos $\mathrm{K}$ e Ca, que estão presentes nas amostras, não foram avaliados nesse estudo, uma vez que estão em concentrações muito elevadas nos reagentes utilizados para simular o sistema gastrointestinal.

Sendo assim, é possível observar na Tabela 4 que a recuperação de todas as amostras ficou entre 81 e $120 \%$, mostrando assim a confiabilidade das análises, uma vez que o somatório das frações foi compatível com a concentração total dos respectivos analitos, conforme já relatado por Souza et al..$^{20}$

Esse estudo permite observar também que em comparação com a concentração total ingerida, apenas uma pequena fração da concentração dos elementos torna-se passível de ser absorvida e utilizada pelo organismo. O elemento $\mathrm{Fe}$, por exemplo, que tem uma grande importância nutricional, teve sua fração bioacessível abaixo do $\mathrm{LD}_{(\mathrm{m})}$ e uma recuperação próxima de $100 \%$ na fração não bioacessível, ou seja, a que simula a excreção pelo organismo.

É possível observar também que as concentrações dos elementos liberados não variaram de forma significativa entre as cultivares, mas os elementos $\mathrm{Ba}, \mathrm{Cu}$ e $\mathrm{Mn}$ apresentaram maiores concentrações bioacessíveis na cultivar Tannat, em comparação com a cultivar Cabernnet Sauvignon. Em contrapartida, o Zn apresentou a maior concentração bioacessível na amostra Cabernet.

Diversos fatores podem ser responsáveis pela não liberação dos nutrientes presentes nesta matriz, dentre eles a quantidade de taninos hidrolisáveis presentes na casca, ${ }^{37}$ os quais complexam com facilidade com íons metálicos e tendem a reduzir a capacidade de liberação e absorção do analito. ${ }^{38,39}$

Outro fator possível de interferir nesse processo é a presença de grupos fosfatos, os quais ocorrem naturalmente em frutas, como na polpa da uva ${ }^{40}$ prejudicando a liberação de elementos para serem absorvidos pelo sistema gastrointestinal pela formação de quelatos..$^{30,41}$ Além disso, as enzimas amilase, tripsina e pepsina são inibidas e/ou prejudicadas pela interação por grupos fosfatos com as proteínas, os 
Tabela 4. Concentração total (CT), concentração da fração bioacessível (FB) e não bioacessível (FNB) em mg kg-1 dos analitos e a recuperação para avaliação da exatidão nas amostras de casca de uva obtidas por MIP OES

\begin{tabular}{|c|c|c|c|c|c|c|}
\hline \multicolumn{7}{|c|}{ Tannat } \\
\hline Analito & $\mathrm{CT}$ & FB & FNB & $\% \mathrm{FB}$ & $\%$ FNB & REC. \\
\hline $\mathrm{Ba}$ & $5,72 \pm 0,22$ & $0,797 \pm 0,026$ & $3,96 \pm 0,04$ & 13,9 & 69,5 & 83,4 \\
\hline $\mathrm{Cu}$ & $3,59 \pm 0,15$ & $1,17 \pm 0,07$ & $2,78 \pm 0,04$ & 32,6 & 77,4 & 110 \\
\hline $\mathrm{Fe}$ & $5,9 \pm 0,3$ & $<0,208$ & $5,55 \pm 0,4$ & ------ & 92,6 & 92,6 \\
\hline $\mathrm{Mn}$ & $2,21 \pm 0,20$ & $0,753 \pm 0,06$ & $1,66 \pm 0,06$ & 43,7 & 75,1 & 118,8 \\
\hline $\mathrm{Pb}$ & $0,51 \pm 0,3$ & $0,133 \pm 0,004$ & $0,41 \pm 0,03$ & 25,5 & 80,4 & 105,9 \\
\hline $\mathrm{Zn}$ & $1,72 \pm 0,03$ & $0,33 \pm 0,006$ & $1,36 \pm 0,06$ & 19,2 & 80,0 & 99,2 \\
\hline \multicolumn{7}{|c|}{ Cabernet Sauvignon } \\
\hline Analito & $\mathrm{CT}$ & FB & FNB & $\% \mathrm{FB}$ & $\%$ FNB & REC. \\
\hline $\mathrm{Ba}$ & $2,36 \pm 0,07$ & $0,32 \pm 0,012$ & $1,74 \pm 0,10$ & 13,5 & 73,9 & 87,4 \\
\hline $\mathrm{Cu}$ & $2,39 \pm 0,20$ & $0,794 \pm 0,007$ & $1,47 \pm 0,13$ & 32,2 & 61,8 & 94,0 \\
\hline $\mathrm{Fe}$ & $5,5 \pm 0,5$ & $<0,208$ & $5,65 \pm 0,4$ & ------ & 102,7 & 102,7 \\
\hline $\mathrm{Mn}$ & $1,92 \pm 0,07$ & $0,67 \pm 0,06$ & $1,26 \pm 0,06$ & 34,9 & 65,6 & 100,5 \\
\hline $\mathrm{Pb}$ & $0,25 \pm 0,01$ & $0,132 \pm 0,013$ & $0,15 \pm 0,11$ & 52,8 & 60,0 & 112,8 \\
\hline $\mathrm{Zn}$ & $3,35 \pm 0,19$ & $0,453 \pm 0,023$ & $2,27 \pm 0,11$ & 13,5 & 67,6 & 81,1 \\
\hline
\end{tabular}

Média \pm desvio padrão.

quais são responsáveis por promover a ligação com o elemento, o que desfavorece sua liberação. ${ }^{42,43}$

A ação das fibras insolúveis que estão presentes em cascas de alimentos também pode interferir no processo de liberação, uma vez que aceleram o tempo do trânsito intestinal, carreando junto os minerais e diminuindo a possibilidade de absorção. ${ }^{26}$

\section{CONCLUSÕES}

Com base nos resultados obtidos foi possível verificar a adequabilidade do método empregado para a determinação de metais em amostras de casca de uva por MIP OES. O preparo das amostras por decomposição ácida com sistema de refluxo apresentou boa precisão e exatidão. Através deste estudo foi possível verificar que diferentes cultivares, mesmo estando no mesmo solo, apresentam diferentes níveis de concentração elementar na casca de uva.

Em relação à fração bioacessível, observou-se que a concentração dos elementos liberados não variou de forma significativa entre as cultivares, e também que a quantidade liberada para absorção pelo organismo não é suficiente para a necessidade diária desses elementos, sendo a casca de uva um subproduto que apresenta potencial para ser consumido de forma complementar na dieta, através da sua inserção em outros alimentos ou na fabricação destes. Os elementos considerados não essenciais, como $\mathrm{Ba} \mathrm{e} \mathrm{Pb}$, mostraramse bioacessíveis, principalmente o $\mathrm{Pb}$ que apresentou valores acima do recomendado pela legislação vigente. Embora esse valor esteja acima do recomendado, não significa que será todo absorvido pelo organismo. Sendo assim, é necessário monitorar a presença desses e de outros elementos para que se tenha um maior controle dos níveis de contaminantes inorgânicos em alimentos, considerando sua posterior aplicação em diferentes produtos.

\section{MATERIAL SUPLEMENTAR}

As Tabelas de condições operacionais de trabalho, concentração dos reagentes utilizados nas soluções do sistema digestivo, parâmetros de mérito para determinação da concentração total e fração bioacessível dos analito, resultados obtidos do teste de adição e recuperação da concentração total e avalição da exatidão do método de preparo de amostra proposto por comparação com sistema fechado de alta pressão, podem ser obtidas como material suplementar em http://quimicanova.sbq.org.br, com acesso livre.

\section{AGRADECIMENTOS}

Os autores agradecem ao apoio financeiro da Coordenação de Aperfeiçoamento de Pessoal de Nível Superior - Brasil (CAPES, Código Financeiro 001), ao Conselho Nacional de Pesquisa e Desenvolvimento Tecnológico (CNPq - Processo $n^{\circ} 422391$ / 2018-2), ao Instituto Nacional de Ciência e Tecnologia Analítica Avançada (INCTAA) (CNPq - Processo n ${ }^{\circ} 573894$ / 2008-6 e Processo FAPESP n ${ }^{\circ} 2008$ / 57808-1) e as bolsas concedidas pelos órgãos de fomento CNPq, bolsa PQ (ASR) (Processo no 305059 / 2019-0) e pela CAPES.

\section{REFERÊNCIAS}

1. Makris, D. P.; Andrikopoulos, N. K.; J. Food Compos. Anal. 2007, 20, 125

2. Cataneo, C. B.; Caliari, V.; Gonzaga, L. V.; Kuskoski, E. M.; Fett, R.; Semina: Ciências Agrárias 2008, 29, 93.

3. Dwyer, K.; Hosseinian, F.; Rod, M.; J. Food Res. 2014, 3, 91.

4. Kammerer, D.; Claus, A.; Carle, R.; Schieber, A.; J. Agric. Food Chem. 2004, 52, 4360.

5. Murga, R.; Ruiz, R.; Beltrán, S.; Cabezas, J. L.; J. Agric. Food Chem. 2000, 48, 3408

6. Yilmaz, Y; Toledo, R. T.; J. Food Compos. Anal. 2006, 19, 41.

7. Deng, Q.; Penner, M. H.; Zhao, Y.; Food Res. Int. 2011, 44, 2712.

8. Piovesana, A.; Bueno, M. M.; Klajn, V. M.; Braz. J. Food Technol. 2013, 16,68 .

9. Paz, M. F.; Marques, R. V.; Schumann, C.; Corrêa, L. B.; Corrêa, É. K.; Braz. J. Food Technol. 2015, 18, 128.

10. Oliveira, R. M.; Oliveira, F. M.; Hernandes, J. V.; Jacques, A. C.; Revista do Congresso Sul Brasileiro de Engenharia de Alimentos 2016, 2, 1. 
11. Machado, T. O. X.; Guedes, T. J. F. L.; Ferreira, T. O.; Melo, B. C. A.; Brazilian Journal of Development 2020, 6, 19260.

12. Tournour, H. H.; Segundo, M. A.; Magalhaes, L. M.; Barreiros, L.; Queiroz, J.; Cunha, L. M.; Ind. Crop. Prod. 2015, 4, 397.

13. http://www.ibravin.org.br/Dados-Estatisticos, acessada em Janeiro 2021.

14. Ahmadi, S. M.; Ali siahsar, B.; Cienc. Invest. Agrar. 2011, 38, 291.

15. Rockenbach, I. I.; Gonzaga, L. V.; Rizelio, V. M.; Gonçalves, A. E. S. S.; Genovese, M. I.; Fett, R.; Food Res. Int. 2011, 4, 897.

16. Pereira, C. C.; Do Nascimento Da Silva, E.; De Souza, A. O.; Vieira, M. A.; Ribeiro, A. S.; Cadore, S.; J. Food Compos. Anal. 2018, 68, 73.

17. Pinto, A. M.; Boeira, A. C.; Lisboa, M.; Medina, A.; Ribeiro, A. S.; Vieira, M. A.; J. Braz. Chem. Soc. 2019, 30, 2395.

18. Sampaio, M. F.; Lisboa, M. T.; Timm, J. G.; Ribeiro, A. S.; Otero, D. M.; Zambiazi, R. C.; Vieira, M. A.; Anal. Methods. 2020, 12, 1360.

19. Cozzolino, S. M. F.; Biodisponibilidade de Nutrientes, 5 ed., Manole: São Paulo, 2016.

20. Souza, A. O.; Pereira, C. C.; Heling, A. I.; Quadro, O. E.; Cadore, S.; Ribeiro, A. S.; Vieira, M. A.; J. Food Compos. Anal. 2019, 77, 60.

21. Minekus, M.; Alminger, M.; Alvito, P.; Ballance, S.; Bohn, T.; Bourlie, C.; Carrrière, F.; Boutrou, R.; Corredig, M.; Dupont, D.; Dufour, C.; Egger, L.; Golding, M.; Karakaya, S.; Kirkhus, B.; Le feunteun, S.; Lesmes, U.; Marcierzanka, A.; Mackie, A.; Marze, S.; Mcclements, D. J.; Ménard, O.; Recio, I.; Santos, C. N.; Singh, R. P.; Vegarud, G. E.; Wickham, M. S. J.; Weitschies, W.; Brodkorb, A.; Food Funct. 2014, 5, 1113.

22. Schmite, B. F. P.; Bitobrovec, A.; Hacke, A. C. M.; Pereira, M. P.; Weinert, P. L.; Anjos, V. E. Food Chem. 2019, 281, 285.

23. Menezes, E. A.; Oliveira, A. F.; França, C. J.; Souza, G. B.; Nogueira, A. R. A. Food Chem. 2018, 240, 75.

24. Afonso, C.; Cardoso, C.; Ripol, A.; Varela, J.; Quental-Ferreira, H.; Pousão-Ferreira, P.; Ventura, M. S.; Delgado, I. M.; Coelho, I.; Castanheira, I.; Bandarra, N. M. Food Res. Int. 2018, 105, 271.

25. de Andrade, C. K.; de Andrade, J. K.; dos Anjos; V. E.; Quináia, S. P. J. Braz. Chem. Soc. 2019, 30, 2721.
26. Lopes, E. R.; Medina, A. L.; Brandalise, J. N.; Ribeiro, A. S.; Nunes, A. M.; Quim. Nova 2017, 40, 1009.

27. Diniz, L. M. N.; Carrasco, T. S.; Medina, A. L.; Ribeiro, A. S.; Nunes, A. M.; Quim. Nova 2017, 40, 711.

28. Leão, P. R. P.; Medina, A. L.; Vieira, M. A.; Ribeiro, A. S.; Braz. J. Food Technol. 2018, 21, e2017062.

29. Oreste, E. Q.; De Jesus, A.; De Oliveira, R. M.; Da Silva, M. M.; Vieira, M. A.; Ribeiro, A. S.; Microchem. J. 2013, 109, 5.

30. Mahan, L. K.; Scott-stump, S.; Raymond, J. L.; Alimentos, Nutrição e Dietoterapia, 13 ${ }^{\mathrm{a}}$ ed., Elsevier: Rio de Janeiro, 2012.

31. Ko, B. G.; Vogeler, I.; Bolan, N. S.; Clothier, B.; Green, S.; Kennedy, J.; Sci. Total Environ. 2007, 388, 35.

32. Mpelasoka, B. S.; Schachtman, D. P.; Treeby, M. T.; Thomas, M. R.; Aust. J. Grape Wine Res. 2003, 9, 154.

33. Yang, N.; Sun, H.; Encyclopedia of Environmental Health 2011, 1, 414.

34. Kirkby, E. A.; Römheld, V.; International Plant Nutrition Institute 2007, $18,1$.

35. BRASIL. ANVISA. RDC $\mathrm{n}^{\circ} 42$, de 29 de agosto de 2013.

36. WHO/FAO; World Health Organization/Food and Agriculture Organization, Geneva, Technical Report Series, n. 916, 2003.

37. Cerbaro, D.; Rombaldi, C, V.; Sainz, R. L.; Nobre, G, A.; J. Bioen. Food Sci. 2016, 3, 149 .

38. Mueller-harvey, I.; Mcallan, A. B.; J. Plant Biochem. Biotechnol. 1992, 1,151 .

39. Kruger, J.; Taylor, J. R.; Du, X.; de Moura, F. F.; Lönnerdal, B.; Oelofse, A.; Food Chem. 2013, 141, 1019.

40. Borges, E. P.; A B C Ilustrado da Vinha e do Vinho, $2^{\text {a }}$ ed., Ed. Mauad: Rio de Janeiro, 249 p, 2008.

41. Ou, K.; Cheng, T.; Xing, Y.; Lin, L.; Nourt, R.; Liang, J.; J. Food Sci. Technol. 2011, 48, 598.

42. Leal, A. S.; Gonçalves, C. G.; Vieira, I. F. R.; Cunha, M. R. R.; Gomes, T. C. B.; Marques, F. R.; Nutrire Rev. Soc. Bras. Aliment. Nutr. 2010, 35, 39.

43. Benevides, C. M. J.; Souza, M. V.; Souza, R. D. B.; Lopes, M. V.; Segurança Alimentar e Nutricional 2011, 18, 67. 